\title{
DELIMITACIÓN DE LA RONDA HÍDRICA CON CRITERIO ECOSISTÉMICO DEL RÍO MIJITAYO, MUNICIPIO DE PASTO-NARIÑO
}

\author{
DELIMITATION OF THE ROUND RIVER WITH ECOSYSTEMIC \\ CRITERIA OF THE MIJITAYO RIVER, PASTO MUNICIPALITY-NARIÑO
}

\author{
Laura Inés Bravo-Sossa ${ }^{1}$ \\ Lizeth Yuranny Romo-Bravo ${ }^{2}$ \\ Sandra Milena Madroñero-Palacios ${ }^{3}$ \\ Francisco Ricardo Mafla-Chamorro ${ }^{4}$ \\ ${ }^{1,2}$ Ing. Ambiental. Universidad Mariana. San Juan de Pasto, Colombia \\ ${ }^{3}$ MSc Manejo Integrado de Cuencas Hidrográficas. Universidad Mariana. San Juan de Pasto, Colombia \\ ${ }^{4}$ MSc en Ingenieria Mención Recursos hídricos. Universidad Mariana. San Juan de Pasto, Colombia

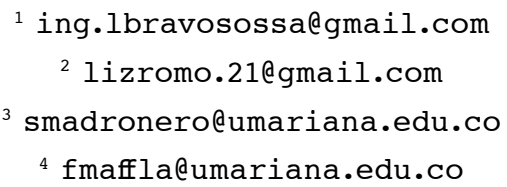

\section{RESUMEN}

El estudio se desarrolló en el río Mijitayo, ubicado en el Municipio de Pasto, suroccidente colombiano. El objetivo fue aplicar una metodología basada en la protección de los ecosistemas para definir el ancho de ronda hídrica y las especies más apropiadas para su recuperación, teniendo como principal elemento la identificación de zonas de vida que se encuentran en el área de estudio. Para ello, se estudiaron algunas de las características fitosociológicas entre las cuales se encuentran: altura, cobertura, densidad y frecuencia relativa, que sirvieron para determinar el Índice de Valor de Importancia (IVI), parámetro fundamental junto con la densidad de drenaje y el área de la cuenca aferente para la delimitación de la ronda hídrica con criterios ecosistémicos. En el sitio de estudio se encontraron las zonas de vida Bosque seco montano bajo (bs-MB), Bosque húmedo montano (bh-M), Bosque muy húmedo montano (bmh-M) y Páramo sub andino ( $\mathrm{p}-\mathrm{SA}$ ) estimando un ancho de ronda hídrica de 15, 25, 29 y 23 metros, respectivamente. Finalmente, las especies recomendadas para su recuperación fueron Verbesina arbórea, seguida de Fuchsia dependens y Bocconia Frutescens para el bs-MB; Tibouchina mollis y Verbesina arbórea para el bh-M; 
Baccharis latifolia seguida de Maytenus prunifolia y Cavendishia bracteata para el bmh-M y por último, Phyllanthus Salviifolius seguida de Munnozia Sp para el p-SA.

Palabras clave: Ronda Hídrica, cuenca, índice de valor de importancia.

\section{ABSTRACT}

The study was conducted in Mijitayo river, which is located in Pasto municipality, in the south-west of Colombia. The general objective was to apply a methodology based in the ecosystems protection to define round river width and the more suitable species to its recovery, having as main element the life zones identification that are in the study area. With this purpose, some of the phytosociological characteristics were studied, among which are: height, coverage, density and relative frequency, which are useful to determine the
Importance Value Index (IVI), fundamental parameter along with the drainage density and the area of the afferent basin.

The following life zones were obtained in the study site: the lower montane dry forest (bs$M B)$, the humid montane forest (bh-M), the very montane forest (bmh-M) and the sub-Andean Paramo ( $\mathrm{p}-\mathrm{SA}$ ) with an estimated round river width of 15, 25, 29 and 23 meters, respectively. Finally, the recommended species for recovery were Verbesina arbórea, followed by Fuchsia dependens and Bocconia Frutescens for bs-MB; Tibouchina mollis and Verbesina arbórea for bh-M; Baccharis latifolia followed by Maytenus prunifolia and Cavendishia bracteata for bmh-M and finally, Phyllanthus Salviifolius followed by Munnozia $S p$ for $\mathrm{p}-\mathrm{SA}$.

Key words: Water round, basin, Importance Value Index.

\section{INTRODUCCIÓN}

Una cuenca hídrica se considera como un área geográfica donde se moviliza un sistema de corrientes de agua a nivel superficial y subterráneo, los cuales confluyen en un mismo punto. En dicha área se propicia la interacción de procesos naturales y actividades humanas. (Formica, et al., 2015).

Como parte integral de los cuerpos de agua se encuentran las zonas de ribera, cuya vegetación se caracteriza por ubicarse en sectores de transición entre hábitats terrestres y acuáticos en sus diferentes estratos (Romero, et al., 2014). Dentro de las funciones de las áreas de ribera se destacan la amortiguación de crecidas y transporte de nutrientes y/o contaminantes, sumado a la protección del ecosistema acuático y de ribera.
Las características bióticas y abióticas se afectan en dichas zonas, principalmente por intervenciones antrópicas, generando un fuerte impacto sobre la calidad del agua y causando graves reducciones de la diversidad biológica (Galeano, et al., 2017). Considerando la problemática expuesta, en este artículo se presenta la aplicación de una metodología basada en la preservación de los ecosistemas en zonas de ribera, bajo la premisa de ser considerados corredores para el establecimiento y tránsito de numerosas especies que migran o se desplazan en su interior, donde se mantienen los requerimientos de su hábitat (García, 2007); y de reguladores de caudales y mantenimiento de condiciones micro-climáticas de dichas zonas (Magdaleno; 2013a). 
La metodología del presente estudio se ejecutó tomando como referencia la normatividad que plantea el mantenimiento de los ríos y los ecosistemas circundantes a estos; el decreto 2245 de 2017 contempla la Guía Técnica de Criterios para el Acotamiento de las Rondas Hídricas en Colombia adoptada por resolución del Ministerio de Ambiente y Desarrollo Sostenible, donde se establece la metodología para el acotamiento de unas zonas de preservación y restauración conocidas como rondas hídricas y afirma que el valor del ancho de protección no debe ser inferior a 30 metros a cada lado del cauce (Minambiente; 2018).

El estudio consistió en determinar el ancho de la ronda del Río Mijitayo por medio del promedio de la altura de los arboles dominantes $(H)$, lo cual se realizó con base en la información obtenida tras la realización de los muestreos y el cálculo del área y la densidad de drenaje de la microcuenca. Finalmente, se propusieron las especies nativas idóneas para dicha ronda a través de características fitosociológicas como la cobertura, densidad y frecuencia relativa con las cuales se determinó el Índice de Valor de Importancia (I.V.I) por cada especie identificada en cada zona de vida.

\section{MATERIALES Y MÉTODOS}

Área de estudio. La presente investigación se realizó en el río Mijitayo, al occidente de la ciudad de Pasto, Departamento de Nariño, Colombia, en las estribaciones del volcán Galeras a $1^{\circ} 13^{\prime}$ Latitud Norte y $77^{\circ} 17^{\prime}$ Longitud Oeste del meridiano de Greenwich (Jiménez, et al., 1989 citado por Madroñero, 2006). Su parte alta está comprendida por bosques y áreas con vegetación herbácea y/o arbustiva, por lo cual los ecosistemas que caracterizan la zona son páramo y sub páramo (CORPONARIÑO; 2009). La parte media presenta bosques densos y fragmentados, se caracteriza por la existencia de un cañón con vertientes cubiertas de eucaliptos y vegetación secundaria. Finalmente, la parte baja del río es considerada como una zona de transición del área rural a la urbana (Jiménez; 1989, Madroñero; 2006).

Para la delimitación del área de estudio se emplearon mapas de ordenamiento de la Subcuenca del río Pasto obtenidos del Plan de Ordenamiento Territorial de Pasto, 2009; en donde se observó la sectorización hídrica del río Mijitayo, con un total de $11.67 \mathrm{Km}^{2}$. Así mismo, presenta cuatro zonas de vida que corresponden a Bosque seco montano bajo (bs-MB), Bosque húmedo montano (Bh-M), Bosque muy húmedo montano (bmh-M) y Páramo sub-andino (p-SA) (CORPONARIÑO; 2009).

Inventario florístico. Se desarrollaron muestreos en un área total de 0,1 ha con el fin de asegurar su representatividad, se establecieron 9 transectos de $2 \times 50 \mathrm{~m}$ cada uno (Mostacedo y Fredericksen; 2000), distribuidos en las cuatro zonas de vida, cada punto establecido fue georreferenciado y en cada transecto se consideraron las especies arbóreas nativas (Minambiente; 2018) mayores a $3 \mathrm{~m}$ de altura y con diámetro a la altura del pecho (DAP) mayor a 2,5 cm (Mateucci y Colma; 1982). Con base en lo anterior, se procedió a determinar un promedio total de alturas de todos los individuos inventariados para determinar la altura de los árboles dominantes $(\mathrm{H})$ por cada zona de vida.

Ancho de la zona de ribera. Ya calculada la altura $(\mathrm{H})$ y el ancho del componente ecosistémico se determinó la relación que existe entre la densidad de drenaje de las corrientes y el área de la cuenca aferente. A partir de esta relación se obtuvo el valor de $\mathrm{N}$, (cuadro 1 ) con el cual se determinó la franja de terreno correspondiente al componente ecosistémico, es decir el ancho de la zona de ribera (Minambiente; 2018). 
Para la determinación de la densidad de drenaje $\left(D_{d}\right)$ de la microcuenca Mijitayo (Ecuación 1) se tuvo en cuenta la Guía Técnica de Criterios para el Acotamiento de las Rondas Hídricas en Colombia (2018). Se emplearon los programas AutoCAD 2015 y Excel 2013, donde se introdujeron los datos obtenidos en campo con relación a longitud de todas las corrientes de agua en kilómetros $(\mathrm{km})$ que se encuentran dentro de la microcuenca Mijitayo y el área de la hoya en kilómetros cuadrados $\left(\mathrm{km}^{2}\right)$.
Para llevar a cabo lo anterior, se aplicó la siguiente ecuación:

\section{$\mathbf{D}_{d}=\frac{L}{A} \quad($ Ecuación 1$)$}

Donde:

$\mathrm{Dd}=$ Densidad de drenaje $\left(\mathrm{km} / \mathrm{km}^{2}\right)$

$\mathrm{L}=$ Longitud total de las corrientes de agua $(\mathrm{km})$

$\mathrm{A}=$ Área total de la hoya $\left(\mathrm{km}^{2}\right)$

Tabla 1. Valor de $N$ según área de la cuenca y densidad de drenaje.

\begin{tabular}{|c|c|c|c|}
\hline \multirow{4}{*}{$\begin{array}{l}\text { Área de cuenca aferente } \\
\left(\mathbf{k m}^{2}\right)\end{array}$} & \multicolumn{3}{|c|}{ Valor de $\mathbf{N}$} \\
\hline & \multicolumn{3}{|c|}{ Densidad drenaje } \\
\hline & Baja & Media & Alta \\
\hline & $<0,5 \mathrm{~km} / \mathrm{km}^{2}$ & $1,0-0,5 \mathrm{~km} / \mathrm{km}^{2}$ & $>1,0 \mathrm{~km} / \mathrm{km}^{2}$ \\
\hline $0<A \leq 1$ & 2,0 & 1,5 & 1,0 \\
\hline $1<A \leq 10$ & 2,5 & 2,0 & 1,5 \\
\hline $10<A \leq 100$ & 3,0 & 2,5 & 2,0 \\
\hline $100<A \leq 1000$ & 3,5 & 3,0 & 2,5 \\
\hline $1.000<A \leq 10.000$ & 4,0 & 3,5 & 3,0 \\
\hline $10.000<A \leq 100.000$ & & 4,0 & \\
\hline
\end{tabular}

Fuente: MADS y UNAL; 2012

Selección de especies arbóreas nativas para la zona de ribera. Inicialmente se determinaron las características fitosociológicas que presentaban los individuos encontrados después de la realización de los muestreos en la zona de estudio (Mateucci y Colma; 1982). (Ecuaciones 2, 3, 4, 5).

Cobertura relativa

$$
C R=\left(\frac{I t}{I e}\right) \times 100 \quad \text { (Ecuación 2) }
$$

Donde:

$\boldsymbol{C R}=$ Cobertura relativa
$\boldsymbol{I} \boldsymbol{e}=$ Sumatoria de intercepción de cada especie

$\boldsymbol{I t}=$ Sumatoria de intercepción de todas las especies

Densidad relativa

Donde:

$\boldsymbol{C} \boldsymbol{R}=$ Densidad relativa

$\mathbf{N}=$ Número de individuos

$\boldsymbol{A}=$ Área en la que se encontraron los individuos 
Frecuencia absoluta

$$
F A=\left(\frac{a i}{U M}\right) \text { (Ecuación 4) }
$$

Donde:

$\boldsymbol{F} \boldsymbol{A}=$ Frecuencia absoluta

$\boldsymbol{a} \boldsymbol{i}=$ Número de apariciones de determinada especie

$\boldsymbol{U M}=$ Unidades muestreales establecidas por zona de vida

Frecuencia relativa

$$
F R=\left(\frac{F A}{A}\right) \times 100 \quad \text { (Ecuación 5) }
$$

Donde:

$\boldsymbol{F} \boldsymbol{R}=$ Frecuencia relativa

$\boldsymbol{F A}=$ Frecuencia absoluta

$\boldsymbol{A}=$ Número de apariciones de todas las especies

Se calculó el Índice de Valor de Importancia, (Ecuación 6) con base en la sumatoria de las variables de las ecuaciones 3,4 y 5 que representan las características fitosociológicas, tales como la cobertura relativa, densidad relativa y frecuencia relativa (Mostacedo y Fredericksen; 2000).

Índice de valor de importancia

$\boldsymbol{I V I}=\boldsymbol{C R}+\boldsymbol{D} \boldsymbol{R}+\boldsymbol{F} \boldsymbol{R}($ Ecuación 6)

Donde,

$\boldsymbol{C} \boldsymbol{R}=$ Cobertura relativa

$\boldsymbol{D} \boldsymbol{R}=$ Densidad relativa

$\boldsymbol{F} \boldsymbol{R}=$ Frecuencia relativa

\section{RESULTADOS Y DISCUSIÓN.}

Inventario florístico. A partir de los muestreos desarrollados en las zonas de vida se identificaron 1018 individuos (Ver ANEXO 1), distribuidos en 20 familias, entre las más abundantes se destacan Asteraceae y Melastomataceae, entre las especies más representativas 26 especies, entre las cuales se encuentran Baccharis Latifolia (Chilca), Verbesina arbórea (Velo), Tibouchina mollis (Flor de Mayo), Otholobium mexicanum (Tarta), Cavendishia bracteata, Fuchsia dependens (Zarcillejo), etc. (Tabla 2).

Tabla 2. Individuos registrados por cada zona de vida.

\begin{tabular}{l|c|c} 
Zona de vida & No individuos nativos & No de transectos \\
\hline $\begin{array}{l}\text { Bosque seco montano bajo (bs-MB). } \\
2750 \text { - } 2927 \text { m s. } \mathrm{n} . \mathrm{m} .\end{array}$ & 122 (6 especies y 5 familias) & 2 \\
\hline $\begin{array}{l}\text { Bosque húmedo montano (Bh-M) } \\
2961-3099 \text { m s. } \mathrm{n} . \mathrm{m} .\end{array}$ & 362 (17 especies y 14 familias) & 2 \\
\hline $\begin{array}{l}\text { Bosque muy húmedo montano (bmh-M) } \\
3450 \text { - } 3575 \text { m s. } \mathrm{n} . \mathrm{m} .\end{array}$ & 388 (24 especies y 18 familias) & 4 \\
\hline $\begin{array}{l}\text { Páramo sub-andino (p-SA) } \\
\text { m.s.n.m. }\end{array}$ & 146 (2 especies y 10 familias) & 1 \\
\hline
\end{tabular}

En el Bosque seco montano bajo, se evidenció que la diversidad en relación con la vegetación arbórea nativa es menor en comparación con las demás zonas de estudio, lo cual se rectifica con observaciones ejecutadas tras inventarios florísticos levantados en la zona (Jiménez y Andrade; 2015). Así mismo y tras la influencia de otros factores como son las áreas inundables a 
lo largo del cuerpo hídrico, la situación descrita, principalmente podría deberse a que en estos ecosistemas se han desarrollado procesos de intervención antrópica principalmente de carácter agrícola y ganadero (Solarte et al. 2007).

La familia más abundante fue Asteraceae con dos tipos de especies, Verbesina arbórea (Velo) y Baccharis latifolia (chilca). Usando como referente el inventario florístico del sector de Buga Alto, del bosque de Paquiestancia, Cayambe - Ecuador, se encontró en común la presencia de la especie Baccharis latifolia en la misma zona de vida, el área en donde se realizó dicho inventario se encuentra a una altura de 2830 m s. n. m (Gutiérrez; 2010). El anterior caso es similar a la presente investigación, por lo que se puede afirmar que las condiciones ambientales mencionadas propician el desarrollo de este tipo de especie.

Con respecto al bosque húmedo montano, a diferencia de la primera zona de vida, se encontró mayor diversidad de especies arbóreas nativas debido a que al presentar una altitud considerable, el acceso es más limitado y por ende la actividad antrópica tiende a decrecer. La familia más abundante fue Melastomataceae con la especie Tibouchina mollis (Flor de mayo), que obtuvo una cifra significativa de individuos en comparación con las demás especies encontradas.

Lo anterior se logra comprobar con los resultados del inventario florístico realizado por la Universidad de Nariño, cuyo objeto fue determinar la capacidad de adaptación y crecimiento de diferentes tipos de especies nativas en la granja Botana localizada a $7 \mathrm{~km}$ de la ciudad de Pasto a una altura de 2970 m.s.n.m, arrojando como resultado final a Tibouchina mollis como el individuo más apto para implementarse en una reforestación, gracias a su rápido crecimiento (Luteyn; 2007).
Posteriormente, en el bosque muy húmedo montano la diversidad con respecto a especies arbóreas nativas fue mayor, encontrando que a medida que aumentaba la altitud, la altura de los arboles disminuía, en comparación con las dos zonas de vida que antecedieron. Esto puede ser causado debido a condiciones climáticas como la temperatura, que en esta área es baja.

También se identificó que la familia más abundante fue Asteraceae con especies como Baccharis latifolia (Chilca) y Verbesina arborea (Velo). Se logró contrastar la información mencionada con un estudio de biodiversidad de especies arbóreas en el (bmh-M) realizado en las cuencas altas de los ríos Pasto y Guamués dentro del municipio de Pasto, en el cual, una de las especies más abundantes fue Baccharis latifolia (Ordoñez et al. 2014).

Finalmente, en el Páramo sub-andino, los elementos climáticos como la humedad cambiaron en comparación con las anteriores zonas, esto se debe a una relación inversamente proporcional, debido a que, a mayor altitud, la presión disminuye, influyendo directamente sobre el componente mencionado, además en zonas donde hay presiones bajas, dicho factor aumenta (Faasafety; 2008). Esto explica el cambio de características generales en la última zona de vida, puesto que se observó la presencia de adaptaciones en el ecosistema, representadas en vellosidades en las hojas y tallos de plantas, el suelo presentaba mayor humedad, acompañada de musgos, helechos y abundante hojarasca. Además, se encontraron plantas epífitas que crecían sobre los árboles. En lo que se refiere a la altura, las especies arbóreas nativas eran más bajas a diferencia de las encontradas en las primeras zonas muestreadas, sin embargo, la altura de los individuos muestreados estuvo entre los 9 y los 22 metros. 
La familia con mayor abundancia fue Asteraceae con tres tipos de especies en el siguiente orden, Munnozia sp, Verbesina arbòrea (Velo) y Baccharis odorata, en relación con este resultado, en el Plan de Manejo Santuario de Flora y Fauna Galeras, se encuentra un inventario florístico realizado, a partir del cual, se logró la caracterización de diferentes tipos de árboles nativos en donde predominaba Munnozia sp que hace parte del ecosistema de páramo (López et al. 2005).

\section{Establecimiento del ancho de la zona de}

ribera. Por su parte, los valores correspondientes al ancho de la zona de ribera fueron para el Bosque seco montano bajo (bs-MB) de $15 \mathrm{~m}$, para el Bosque húmedo montano (bh-M) de $25 \mathrm{~m}$, para el caso del Bosque muy húmedo montano (bmh-M) el valor es $29 \mathrm{~m}$ y finalmente, para el Páramo sub andino ( $p-S A)$ el valor es de $23 \mathrm{~m}$. Tales anchos se calcularon a partir de la altura promedio de los árboles dominantes $(\mathrm{H})$ muestreados que es de $10 \mathrm{~m}, 17 \mathrm{~m}, 20$ $\mathrm{m}$ y $15 \mathrm{~m}$, correspondientes a una de las zonas de vida listadas anteriormente (figura 1). Cabe resaltar que entre los cálculos relacionados con la geomorfología de la microcuenca Mijitayo, se encuentran el área de la hoya con un valor de $9,87 \mathrm{~km}^{2}$, mientras que el valor referente a la densidad de drenaje $\left(D_{d}\right)$ fue de $2,7 \mathrm{Km} / \mathrm{Km}^{2}$, lo cual indica que se encuentra dentro del rango que usualmente toma valores entre 0,5 para hoyas con drenaje pobre hasta 3,5 para hoyas excepcionalmente bien drenadas (Monsalve, 1999).

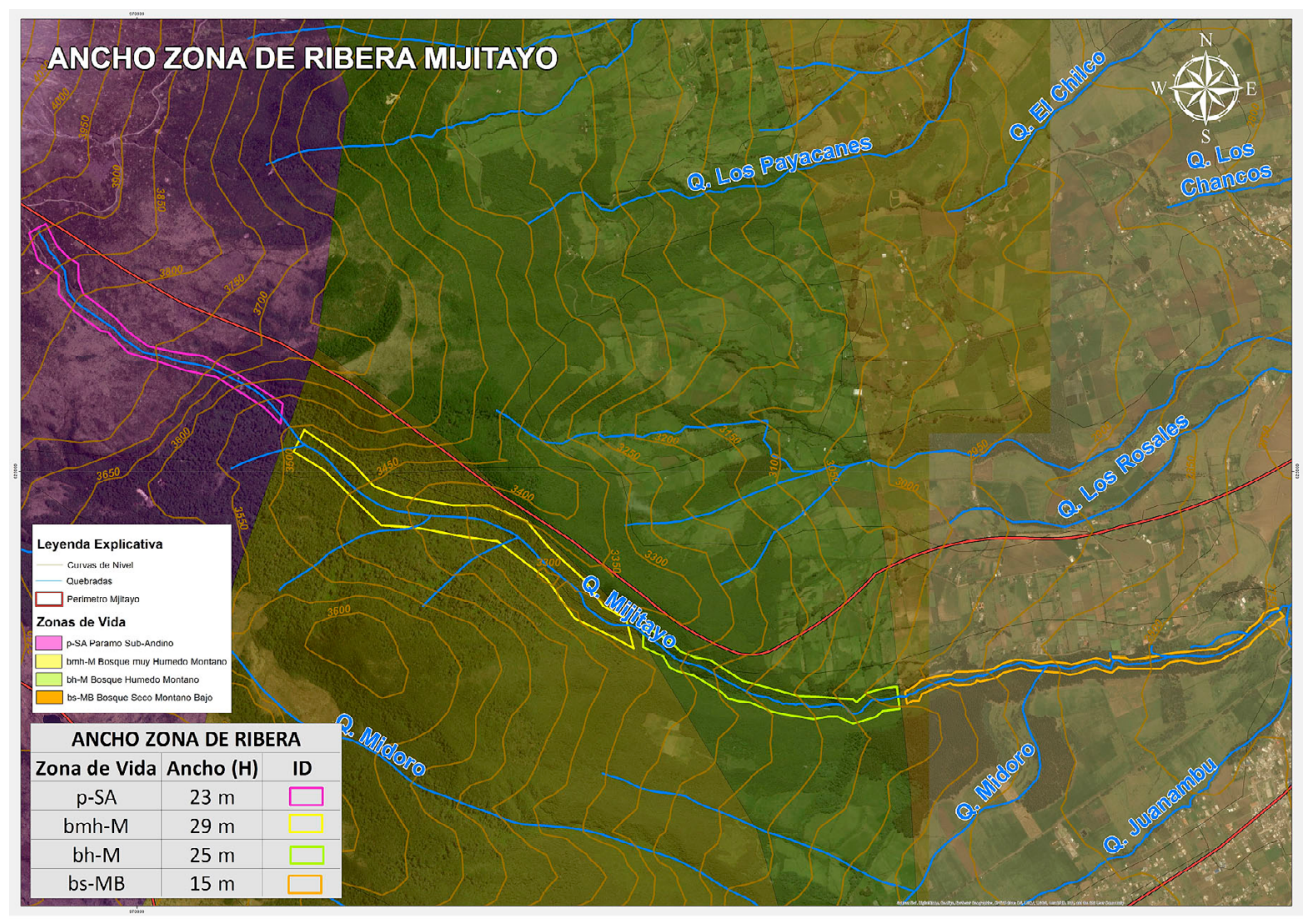

Figura 1. Ancho de la zona de ribera para cada zona de vida.

Con lo anterior se identificó el valor de $\mathrm{N}$ que fue de 1,5 adimensional (tabla 1) y se operó con el valor de $\mathrm{H}$ para cada zona de vida, obteniendo los anchos de la zona de ribera que se mencionan al inicio de este apartado.
Según lo observado, los valores se vieron influenciados por los procesos de intervención antrópica ya sea deforestación o ampliación de la frontera agrícola y pecuaria que han contribuido a la reducción de la cobertura vegetal 
en la zona de ribera y que por ende, reduce los promedios de las alturas. Es importante establecer que la metodología afirma que entre mayor sea el promedio $\mathrm{H}$, mayor será el ancho de la zona de ribera para cada zona de vida (Minambiente; 2018).

Tras el levantamiento de datos y el cálculo del ancho de la zona de ribera por cada zona de vida muestreada, se identificó que, según la normatividad establecida para ninguno de los casos los valores de ancho llegaron a los 30 metros a partir del nivel medio de aguas máximas que exige la norma colombiana. A partir de ello, inicia una discusión en cuanto a sí dicha dimensión es o no suficiente para garantizar la conservación del recurso hídrico a lo cual (Minambiente; 2018) consideran importante tener en cuenta, además del componente ecosistémico, los componentes hidrológico y geomorfológico, ya que estos son menos variables en periodos cortos de tiempo. Estudios desarrollados por Fundaguiza (2013) en un tramo de los ríos Pasto y Miraflores, muestran cómo la determinación de la zona de ribera es mayor cuando se aplica el análisis geomorfológico e hidrológico.

Sin embargo, y según el objetivo planteado será totalmente diferente el ancho de la zona de ribera, ya que depende de la composición y densidad de la vegetación presente, la longitud y tipo del curso de agua, además de la pendiente del terreno (Fischer y Fischenich; 2000).

Así mismo, la determinación apropiada de los anchos de franjas de protección de cauces debe responder a la función que se espera satisfacer, siendo el enfoque de esta investigación la protección desde una perspectiva de conservación de la vegetación de ribera, sin desconocer que adicionalmente es necesario proveerle a los ríos el espacio para desarrollar otros procesos, tales como: la preservación de los ecosistemas acuáticos, el desarrollo de los procesos de erosión, entre otros (Gayoso y Gayoso; 2003).

El manejo de la zona de ribera variará de muy estrecho a ancho dependiendo de la geomorfología fluvial en la zona, como lo son las llanuras de inundación, valles, entre otros y también del tipo de uso del suelo adyacente, por ejemplo, un parque nacional, agricultura, silvicultura, vivienda urbana, entre otros (Price y Lovett; 2002).

\section{Propuesta de especies nativas para la restauración ecológica. Finalmente, con} base en los resultados obtenidos en el estudio fitosociológico; se propusieron las especies que presentaron los mayores índices de valor de importancia (IVI), las cuales, se consideran más idóneas para ser implementadas en las cuatro zonas de vida identificadas. Para el Bosque seco montano bajo, fueron Verbesina arbórea y Fuchsia dependens. Por su parte, las especies seleccionadas para el Bosque húmedo montano fueron Tibouchina mollis y Verbesina arbórea. En el caso del bosque muy húmedo montano, se seleccionó Baccharis latifolia y Maytenus prunifolia; por último, las especies elegidas para el p-SA, fueron Phyllanthus Salviifolius seguida de Munnozia Sp (Tabla 3). 
Tabla 3. Especies potencialmente adecuadas para restauración ecológica en la zona de ribera.

\begin{tabular}{|c|c|c|}
\hline Zona de vida & Especies & $\begin{array}{l}\text { Índice de Valor de } \\
\text { Importancia (IVI) más altos }\end{array}$ \\
\hline \multirow{2}{*}{$\begin{array}{l}\text { Bosque seco montano bajo (bs-MB). } \\
2750 \text { - } 2927 \text { m s. } n . \text { m. }\end{array}$} & Verbesina arbórea & 78,4 \\
\hline & Fuchsia dependens & 55,7 \\
\hline \multirow{2}{*}{$\begin{array}{l}\text { Bosque húmedo montano (Bh-M) } \\
2961 \text { - } 3099 \text { m s. n. m. }\end{array}$} & Tibouchina mollis & 76,4 \\
\hline & Verbesina arbórea & 66,5 \\
\hline \multirow{2}{*}{$\begin{array}{l}\text { Bosque muy húmedo montano (bmh-M) } \\
3450-3575 \text { m s. n. m. }\end{array}$} & Baccharis latifolia & 43 \\
\hline & Maytenus prunifolia & 40,2 \\
\hline \multirow{2}{*}{$\begin{array}{l}\text { Páramo sub-andino (p-SA) } \\
3725 \text { m s. n. m. }\end{array}$} & Phyllanthus Salviifolius & 45 \\
\hline & Munnozia Sp & 40,3 \\
\hline
\end{tabular}

Con dicha propuesta se quiere lograr a futuro, la implementación de una herramienta útil para realizar procesos de restauración ecológica en la zona de ribera del río Mijitayo; debido a que tras la ejecución del estudio se determinó que en ninguno de los tramos se respeta el ancho de la ronda hídrica exigida, la cual, es fundamental para la conservación de las características y el estado óptimo de las fuentes hídricas.

De acuerdo con la tabla 3 se recomienda el uso de Verbesina arbórea; estudios afirman la importancia de la implementación de dicha especie en cercas vivas, con el objetivo de proveer hábitats y recursos que incrementen la conectividad del paisaje, lo cual está íntimamente relacionado con la preservación de los ecosistemas aledaños a los recursos hídricos (Navia et al. 2017).

Dentro de los recorridos del presente estudio se identificó la degradación y el deterioro de los ecosistemas presentes en el Bosque seco montano bajo (bs-MB); principalmente debido a que en estas zonas la intervención antrópica es mayor; por lo cual, es adecuado proponer la restauración con especies que garanticen y mejoren las condiciones de los ecosistemas aledaños al río Mijitayo; logrando mantener y mejorar la calidad del mismo.

Otra especie recomendada es Fuchsia dependens (Zarcillejo), gracias a su capacidad de regulación de escorrentía, control de procesos erosivos y protección de cuencas (Castro y Martínez; 2008). Adicionalmente, esta especie se adapta fácilmente en sitios húmedos y generalmente puede ubicarse en zonas aledañas a los arroyos o cuerpos de agua. Es una planta nativa de fácil adaptabilidad al medio y se caracteriza por ser de rápido crecimiento (Caranqui; 2011). Estas características hacen adecuado el uso de esta especie en la recuperación de la ronda hídrica del río Mijitayo; puesto que además de su rápido crecimiento, lograría evitar la llegada de agroquímicos y demás vertimientos que por escorrentía afectan la calidad del río.

Por su parte, una de las especies recomendada para el Bosque húmedo montano es Tibouchina mollis (siete cueros), la cual influye en procesos de revegetación y restauración ecológica integral, usada como una herramienta que busca la reposición de los valores, bienes y servicios ambientales que las comunidades locales han 
perdido (Cabrera et al. 2014). Caso que se presenta en la zona de vida nombrada para el presente estudio, puesto que a lo largo de los recorridos se evidenció fragmentación del bosque nativo a raíz de la siembra de especies maderables como pinos y eucaliptos, por lo tanto, al implementar dicha especie se lograría además de la recuperación del ecosistema, el mantenimiento y regeneración de los sistemas naturales que se desarrollan alrededor.

Finalmente, la Munnozia Sp fue una especie seleccionada en el estudio, por su función protectora de cuencas que asegura el restablecimiento de la funcionalidad del ecosistema (Mora; 2012). Al ser una de las especies recomendada para implementar en el Páramo Sub- Andino; son de suma importancia las funciones descritas, ya que este es uno de los ecosistemas estratégicos que en la actualidad se debe proteger y conservar, por ser zonas donde se encuentra vegetación abundante, además de ser uno de los lugares donde nacen fuentes hídricas.

\section{CONCLUSIONES}

Los valores obtenidos para la ronda hídrica del río Mijitayo con respecto al componente ecosistémico fueron, para el Bosque seco montano bajo (bs-MB) $15 \mathrm{~m}$, para el Bosque húmedo montano (bh-M) $25 \mathrm{~m}$, para el Bosque muy húmedo montano (bm-M) 29 m y para el Páramo sub-andino ( $p-S A) 23 \mathrm{~m}$, mientras que el Decreto 2245 de 2017 establece que la faja paralela a la línea de mareas máximas o a la del cauce permanente de ríos y lagos debe tener un ancho de mínimo $30 \mathrm{~m}$; por lo tanto, se debe adoptar el valor dictado por esta normatividad, y sumado a ello, el área de protección o conservación aferente que corresponde a los componentes geomorfológico y ecosistémico.

De acuerdo con el análisis desarrollado en torno a los Índices de Valor de Importancia (IVI) más altos y a las características morfológicas y funcionales de los individuos muestreados, las especies nativas aptas para recuperar la ronda hídrica del río Mijitayo fueron Verbesina arbórea (Velo), Fuchsia dependens (Zarcillejo), Bocconia frutescens (Trompeto), Tibouchina mollis (Flor de Mayo), Baccharis latifolia (Chilca), Cavendishia bracteata (Chaquilulo), PhyIlanthus salviifolius (Yuco) y Munnozia sp.

La cobertura arbórea nativa que hace parte de las zonas de vida ubicadas en un rango altitudinal entre los 3450 y 3755 m.s.n.m presenta el mejor estado, debido a que las condiciones topográficas hacen difícil la accesibilidad al territorio, y esto ha reducido en gran medida el grado de intervención antrópica.

A la fecha no existe un sustento legal lo suficientemente robusto que limite la intervención en áreas de ribera y junto a las dificultades de recuperar la ronda en zonas que se encuentran intervenidas, generan la necesidad de desarrollar mecanismos que permitan, no solo el determinar la medida de la ronda sino además el hacer viable su aplicación.

\section{LITERATURA CITADA}

Aguirre, Z. Kvist, L. y Sánchez, O. (2006). Bosques secos en Ecuador y su diversidad. Botánica Económica de los Andes Centrales. 162 -187.

Cabrera, M. y Ramirez, W. (2014). Restauración ecológica de los páramos de Colombia. Transformación y herramientas para su conservación. Instituto de Investigación de Recursos Biológicos Alexander von Humboldt (IAvH). Bogota, D.C. Colombia. 296 pp.

Calder, I. Hofer, T. Vermont, S. y Warren, P. (2007). Towards a new understanding of forests and water. Food and Agriculture Organization of the United Nations, Rome. Unasylva 58 (229). 18 p.

Castro, J y Martínez, E. (2008). Formulación del Plan de Gestión Ambiental del municipio de Sibundoy, Putumayo. Tesis. Universidad Tecnológica de Pereira., 185 p. 
Caranqui, J. (2011). Avances taxonómicos y de propagación del genero Fuchsia en Ecuador. Herbario Politécnico del Chimborazo, (CHEP). 44-45.

Corporación Autónoma Regional de Nariño. (2009). Plan de ordenamiento y manejo ambiental subcuenca del río Pasto. San Juan de Pasto, Colombia., $120 \mathrm{p}$.

Federal Aviation Administration. (2008). Density Altitude. 1-4

Fischer, R y Fischenich, C. (2000). Design Recommendations for Riparian Corridors and Vegetated Buffer Strips. Ecosystem Managamenet \& Restoration Research Program. United States of America.

Formica, S. Sacchi, G. Campodonico, V. Pasquini, A y Cioccale, M. (2015). Modelado De Calidad De Agua En Ríos De Montaña Con Impacto Antrópico. Caso De Estudio: Sierra Chica De Córdoba, Argentina. Revista Internacional de Contaminación Ambiental. 31 (4) 327-341.

Fundaguiza (2013). Determinación de la ronda hídrica de $40 \mathrm{~km}$ en los ríos Pasto y Miraflores. Corporación Autónoma Regional de Nariño Corponariño. Pasto. Colombia. 80 p.

Galeano, E. Monsalve, L. Mancera, N. (2017). Evaluación de la Calidad Ecológica de Quebradas Andinas en La Cuenca del Río Magdalena, Colombia. Revista U.D.C.A Actualidad \& Divulgación Científica 20 (2): 413-424

García, C. (2007). Regulación Hídrica Bajo Tres Coberturas Vegetales en la Cuenca del Río San Cristóbal, Bogotá D.C. Revista Colombia Forestal, 10(20), $141 \mathrm{p}$.

Gayoso, J. y Gayoso, S. (2003). Diseño de Zonas Ribereñas. Requerimiento de un Ancho Mínimo. Universidad Austral de Chile: Facultad de Ciencias Forestales. Valdivia, Chile. 12 p.

Gómez, E. Gómez, F. (2009). Revista de Arquitectura. Universidad Católica de Colombia. Facultad de Arquitectura. Volumen 11. Bogotá D.C., Colombia. 8 p. ISSN: 1657-0308.

González del Tánago, M. (2002). Las riberas elementos clave del paisaje y en la gestión del agua. Departamento Ingeniería Forestal. Universidad Politécnica de Madrid. Madrid, España. 13 p.

Gutiérrez, L. (2010). Inventario Florístico del sector de Buga Alto, del Bosque de Paquiestancia, Cayambe - Ecuador. Tesis de pregrado. Universidad Politécnica Salesiana. Cayambe, Ecuador., 115 p.
Green Facts. (2009). Recursos Hídricos. II Informe de las Naciones Unidas Sobre el Desarrollo de los Recursos Hídricos en el Mundo. Bruselas, Bélgica., $6 \mathrm{p}$.

Empresa Metropolitana de Aseo - EMAS S.A E.S.P. (2009). Plan de Ordenamiento y Manejo Ambiental Subcuenca del Rio Pasto. Sectorización hídrica. Mapa $N^{\circ}$ 17. Escala de impresión 1:60.000. Pasto, Colombia.

Jiménez, A. Navas, L. Ortiz, G. y Solarte, A. (1989). Declaratoria de impacto ambiental en las aguas superficiales en la microcuenca del río Mijitayo. Tesis Especialización en Ecología. Universidad de Nariño, Pasto, Colombia., 165 p.

Jiménez, L. Andrade, S. (2015). Caracterización de las cercas vivas y la avifauna asociada en la vereda San Felipe, Microcuenca Mijitayo, Municipio de Pasto, Departamento de Nariño. Universidad de Nariño, Pasto, Colombia, 37 p.

López, N. Pinilla, A. Zambrano, J. Castro, J. Miramag, O. y Pimiento, E. (2005). Plan de Manejo 2006 - 2010 Santuario de Flora y Fauna Galeras. Ministerio de ambiente, Vivienda y Desarrollo Territorial (MAVDT). Pasto, Colombia., 78 p.

Luteyn, J. Buritica, P. (2007). University of Nariño property, vicinity Granja Botana, Nariño. Colombia. Colecciones. Universidad Nacional de Colombia. Recuperado de: http://www.biovirtual. unal.edu.co/ICN/?controlador=ShowObject\&acci on $=$ show $\&$ id $=122837$

Madroñero, S. (2006). Manejo del recurso hídrico y estrategias para su gestión integral en la microcuenca Mijitayo, Pasto Colombia. Tesis Programa de educación para el desarrollo y la conservación. Escuela de posgrado. Centro Agronómico Tropical de Investigación y Enseñanza (CATIE). Turrialba, Costa Rica., 183 p.

Magdaleno, F. (2013). Estructura y composición de la vegetación de ribera. Evolución de los bosques riparios en el Ebro medio. Centro de Estudios de Técnicas Aplicadas (CEDEX), Ministerio de Fomento, Ministerio de Agricultura, Alimentación y Medio Ambiente. Madrid, España., 4 p.

Martínez, C y Fernández, J.A. (2009). Los bosques de ribera. Cartilla forestal. Obra Social Caja Madrid y la Asociación de Forestales de España (PROFOR). Madrid, España, 20-29.

Matteucci, S y Colma, A. (1982). Metodología para el estudio de la vegetación. Secretaría General de la 
Organización de los Estados Americanos: Programa Regional de Desarrollo Científico y Tecnológico. Washington D.C, Estados Unidos., 77 p.

Ministerio de Ambiente y Desarrollo Sostenible. (2018). Guía Técnica de Criterios para el Acotamiento de las Rondas Hídricas. Bogotá D.C., Colombia., $86 \mathrm{p}$.

Monsalve, G. (1999). Hidrología en la Ingeniería. Ed. Escuela Colombiana de Ingeniería. Bogotá D.C, Colombia. 382 p.

Mora, W. (2012). Propuesta de recuperación de la ronda hídrica de un tramo de 2,3 kilómetros en la cuenca alta del rio Dulce, municipio de Sasaima mediante rehabilitación forestal. Tesis. Universidad Libre, Facultad de Ingeniería, Instituto de posgrados. Bogotá D.C., Colombia. 109 p.

Mostacedo, B y Fredericksen, T. (2000). Manual de Métodos Básicos de Muestreo y Análisis en Ecología Vegetal. Proyecto de Manejo Forestal Sostenible Financiado por USAID Y PL480 en convenio con el MDSP. Santa Cruz de la Sierra, Bolivia., 87 p.

Navia, J. Muñoz, D. Solarte, J. (2017). Caracterización del componente arbóreo de cercas vivas en sistemas agroforestales en el departamento de Nariño. TEMAS AGRARIOS - Vol. 22:(2) Julio - Diciembre 2017 (80 - 89). Nariño. Colombia.

Ordoñez, H. Pérez, M. y Quiroz, C. (2004). Estudio de la biodiversidad no cultivada en las cuencas altas de los ríos Guamuéz y Pasto. Revista de ciencias agrícolas. (21) 1 y 2.
Plan de Ordenamiento Y Manejo Ambiental Subcuenca del Río Pasto. (2009) Sectorización hídrica. Mapa No 17. Escala De impresión 1:60.000. Pasto, Nariño.

Presidencia de la República de Colombia. (2016, 11, 12). Decreto Único Reglamentario del Sector Ambiente y Desarrollo Sostenible. [Legislación en formato web]. Recuperado de http://www. alcaldiabogota.gov.co/sisjur/normas/Norma1. jsp? $\mathrm{i}=62511$.

Price, P. y Lovett, S. (2002). Managing riparian land. Land \& water Australia. Fact Sheet 1. Australia. $6 \mathrm{p}$.

Romero, F. Cozano, M. Gangas, R. Naulin, P. (2014). Zonas ribereñas: protección, restauración y contexto legal en Chile. Revista Bosque. 35(1): 3-12.

Solarte, M. Narváez, G. Baca, Elena. Calderón, J. Torres, C. Rengifo, J. Dávila, M. Cepeda, B. Castillo, G. Rivas, G. Muñoz, D. Figueroa, V. Martínez, P. (2007). Proyecto estado del arte de la información biofísica y socioeconómica de los páramos de Nariño. Grupo de investigación en biología de páramos y ecosistemas andinos equipo interdisciplinario proyecto páramos - Universidad de Nariño. Corporación Autónoma Regional de Nariño - Corponariño. Pasto, Nariño., 276 p.

Conflicto de Intereses Los autores declaran no tener ningún conflicto de intereses 